\title{
Results of an Inpatient Smoking Cessation Program: 3-Month Cessation Rate and Predictors of Success
}

Original Article

\author{
Sun-Hee Kim,Jung-Ah Lee, Kye-Un Kim, Hong-Jun Cho* \\ Department of Family Medicine, Asan Medical Center, Ulsan University College of Medicine, Seoul, Korea
}

\section{ABSTRACT}

Background: Hospitalization presents smokers with an opportunity to initiate smoking cessation. We studied the effect of inpatient counseling and follow-up after discharge on smoking cessation and assessed predictors of successful cessation.

Methods: This study included a total of 125 patients (118 male and 7 female) who were admitted to departments of neurology, cardiology, and pulmonology. They were referred to the smoking cessation clinic, and participated between September 2011 and February 2013. A counseling service lasting about thirty minutes was provided by the third-year family medicine residents during hospitalization. The follow-up counseling services, which were either by telephone or in-person physician counseling were provided at 1 week, 4 weeks, and 3 months. Smoking habits and nicotine dependency data were gathered using questionnaires, and patient information was collected from electronic medical records.

Results: The average age in the study was $57.9 \pm 10.2$ years and duration of smoking was $35.9 \pm 11.7$ years. Daily tobacco consumption was $23.5 \pm 13.2$ cigarettes. The smoking cessation rate after 3 months was $42.4 \%$. The only differences between patients in the successful cessation and failed groups were cause of admission ( $\mathrm{P}=0.039)$ and total number of counseling sessions after discharge ( $\mathrm{P}$ $<0.001)$. In a multivariate analysis, smoking cessation was more likely when patients experienced more instances of follow-up after discharge (1-2 visits: odds ratio [OR], 8.186; 95\% confidence interval [CI], 1.060 to 63.239 ; $\geq 3$ visits: OR, $121.873 ; 95 \%$ CI, 14.462 to $1,027.055)$.

Conclusion: Smoking cessation counseling during hospitalization and further follow-up by telephone or outpatient counseling after discharge contributed to an increased smoking cessation rate. The smoking cessation rate also tended to increase with total counseling numbers.

Keywords: Inpatients; Smoking Cessation; Counseling

Received: August 30, 2013, Accepted: December 3, 2014

*Corresponding Author: Hong-Jun Cho

Tel: +82-2-3010-3812, Fax: +82-2-3010-3815, E-mail: hjcho@amc.seoul.kr

Korean Journal of Family Medicine

Copyright (C) 2015 The Korean Academy of Family Medicine

This is an open-access article distributed under the terms of the Creative Commons Attribution Non-Commercial License (http://creativecommons.org/licenses/by-nc/3.0) which permits unrestricted noncommercial use, distribution, and reproduction in any medium, provided the original work is properly cited. 


\section{INTRODUCTION}

Smoking is one of the leading causes of early death, ${ }^{1)}$ creating not only a range of health problems but also an increase in medical care costs. In 2011, World Health Organization (WHO) reported that smoking-related diseases, such as ischemic heart disease, cerebral vessel disease, and lower respiratory disease, were major causes of death globally $(12.9 \%, 11.4 \%$, and $5.9 \%$, respectively). In Korea, the attributable risk of smoking for all male deaths was 30.7\%. Attributable risk of smoking was high for many diseases, for example $26.7 \%$ for ischemic heart disease, $32.2 \%$ for stroke, $72.2 \%$ for lung cancer, and $33.6 \%$ for chronic obstructive pulmonary disease. ${ }^{2)}$ Smoking is known to increase the risk of cancer at various sites other than the lungs. ${ }^{3)}$ There is no doubt that smoking cessation before the occurrence of disease, or abstaining from smoking in the first place, can prevent and reduce mortality. Many recent studies have reported that smoking cessation after the occurrence of disease also has health benefits. ${ }^{4-6)}$

Hospitalization presents smokers with an opportunity to initiate smoking cessation. This opportunity is a good one for several reasons. First, since the entire hospital building is a smoke-free area, inpatient smokers can be freed from smelling tobacco smoke or seeing other smokers. This can decrease the urge to smoke. ${ }^{7)}$ Second, the motivation for inpatient smokers to cease smoking is stronger than for the general population. This is partly because they can be advised to stop smoking by medical workers, and also because just having been diagnosed with a smoking-related disease can affect their motivation to quit. ${ }^{8)}$ Third, they are in a position to learn detailed behavior management strategies for urges and can be prescribed medication to stop smoking by specialized medical workers. As a result, inpatient smokers may have an easier time quitting than smokers who are not hospitalized.

In previous studies, smoking cessation rates a year after inpatient smoking cessation counseling were $14 \%$ to $70 \%$, higher than in the general population. The cessation rate increased with regular follow-ups after discharge. ${ }^{8,9)}$ When inpatient smokers experienced withdrawal symptoms during hospitalization, they were more likely to continue smoking not only in the hospital but also after discharge. ${ }^{10)}$ For these smokers, proper medication and behavioral therapy may be needed to further increase the smoking cessation rate.

While the effectiveness of inpatient smoking cessation counseling has been improving across many studies, a meta-analysis showed that not all counseling programs are effective. ${ }^{8}$ Effective counseling that can influence quitting rates significantly requires sufficient counseling time and regular monitoring after discharge. ${ }^{11-15)}$ There are various opinions about how many follow-up counseling sessions are sufficient, but it is widely reported that monitoring for at least 1 month after discharge is needed. ${ }^{89}$ )

In this study, we provided inpatient smoking cessation counseling and followed up regularly for 3 months after discharge in order to identify the effectiveness of a systematic inpatient smoking counseling program. Patients diagnosed and admitted with smoking-related diseases like cerebral vessel, coronary, and pulmonary disease were included in the study population, so we compared differences in cessation rate by cause of admission. We also considered duration of hospitalization and total number of additional counseling sessions as factors potentially affecting the smoking cessation rate. 


\section{METHODS}

\section{Study Participants}

Inpatient smokers who were admitted to the department of neurology, cardiology, or pulmonology at Asan Medical Center were included in this study. We selected subjects whose doctors had referred them between September 2011 and February 2013 and who had completed the patient's agreement for smoking cessation counseling. A total of 125 patients were included; after the first counseling session, 25 and 33 patients were missed at 4 weeks and 3 months, respectively.

\section{Inpatient Smoking Cessation Program and Follow-Up}

Before smoking cessation counseling, patients were asked to fill out a questionnaire addressing nicotine dependency, age of smoking initiation, duration of smoking, and daily tobacco consumption. A third-year family medicine resident then visited the patient and conducted 30 minutes of intensive education and counseling about smoking cessation at the patient's bedside.

We used 50 Microsoft PowerPoint slides as educational material. The slides contained information about the harm done by smoking, smoking-induced diseases, and the physical, psychological, and economical benefits of quitting smoking. They also explained the physiological reasons for recurring smoking cessation failure, withdrawal symptoms, behavioral management to overcome these challenges, and some pros and cons of each pharmacotherapy. After the educational slides, the doctor confirmed the patient's willingness to quit smoking and asked if they wanted to use pharmacotherapy or to use their own willpower.

We monitored patients at 1 week, 4 weeks, and 3 months after discharge if they agreed to additional follow-up counseling sessions. If patients stayed in the hospital until the first follow-up date, a family medicine resident visited and counseled them again. Nurses trained as professional smoking cessation counselors performed telephone consultations unless a patient wanted to visit the doctor's office. During additional counseling sessions we confirmed whether they had continued to abstain from smoking (and asked how many cigarettes they had smoked in case of failure); we also discussed the advantages and disadvantages of cessation as well as withdrawal symptoms and side effects of pharmacotherapy. If patients had successfully kept up the goal of cessation, doctors gave patients encouragement and compliments to continue their motivation. In case of failure, they were given medical treatment or advice about another attempt at smoking cessation.

\section{Data Collection}

We defined successful smoking cessation as not a single puff from the first counseling date during hospitalization until the present counseling session. We excluded from the study participants who wanted to stop counseling or who died. Participants who could not be reached were considered to have failed to cease smoking, but were not omitted from the study. Nicotine dependency was based 
on the Fagerstrom Test for Nicotine Dependence (FTND) score, which consisted of six questions. ${ }^{16)}$ We used electronic medical records to get basic information about the patients (age, sex, residence, education, drinking, and current marital status), past medical history (hypertension, diabetes mellitus, dyslipidemia, history of coronary disease, and cerebral vessel disease), cause of admission (neurologic, cardiologic, or pulmonologic disease), and duration of hospitalization ( $\leq 3$ days, 4-7 days, or $\geq 8$ days). Nicotine dependency (FTND score: $\geq 7,4-6$, or $\leq 3$ ) and smoking habits (age of initiation, duration, and daily consumption) were collected using self-report questionnaires. We considered patients to have used pharmacotherapy only when they were prescribed medication by a doctor during hospitalization or at discharge. Three medications were used for the study: nicotine patch or gum, varenicline, and slow release bupropion. The total number of counseling sessions $(0,1-2$, or $\geq 3)$ and visits to an outpatient clinic were confirmed by electronic medical records. The first counseling session during hospitalization was not included in the total number of sessions, but sessions conducted with a patient's family members were included.

\section{Statistical Analysis}

To identify differences between the successful group and the failing group, we performed univariate analyses with chi-square tests for education, cause of admission, duration of hospitalization, total number of counseling sessions, visits to an outpatient clinic, FTND score, and use of pharmacotherapy. We used t-tests for age, age of smoking initiation, duration of smoking habit, and daily consumption. We performed a multivariate analysis to identify the factors predicting 3 months of cessation. Statistical analyses were conducted using PASW SPSS ver. 18.0 (SPSS Inc., Chicago, IL, USA) for the univariate analyses and SAS ver. 9.3 (SAS Institute Inc., Cary, NC, USA) for the multivariate analysis. A significance level of $\mathrm{P}<0.05$ was used.

\section{RESULTS}

\section{Baseline Characteristics}

The mean age of participants was $57.9 \pm 10.2$ years. The distribution of education level was relatively even: $32.8 \%$ middle school or less, $36.0 \%$ high school, and $31.2 \%$ college or more. The average age of smoking initiation was $21.1 \pm 4.8$ years, and average duration of smoking was 35.9 \pm 11.7 years. The mean daily consumption was $23.5 \pm 13.2$ cigarettes. The largest percentage of participants (44.8\%) had a nicotine dependence score of 4 to 6 , while $37.6 \%$ scored higher than 7 and $17.6 \%$ scored 3 or less. Doctors prescribed medication for $65.6 \%$ of our participants; the rest relied on education and counseling only.

A total of 125 patients (118 males and 7 females) from the departments of neurology, cardiology, and pulmonology were included during the study recruitment period. There were 53 patients $(42.4 \%)$ from 
neurology, 47 (37.6\%) from cardiology, and 25 (20.0\%) from pulmonology. In terms of diagnosis at admission, cerebral infarction accounted for $94.3 \%$ of the neurology patients and coronary heart disease accounted for $72.3 \%$ of cardiology patients. In the pulmonology department, $32 \%$ of patients had lung cancer, $24 \%$ had chronic obstructive pulmonary disease, and $8 \%$ had pneumonia.

\section{Smoking Cessation Rate}

There were 66 patients who reported successful smoking cessation at 4 weeks after inpatient counseling. Thirty-four patients reported that they did not succeed, and 25 patients were no longer in contact after 4 weeks. Therefore, a total of 59 patients were considered to have failed and the smoking cessation rate was $52.8 \%$. At the 3 -month counseling session, 53 patients reported success, we lost contact with 39, 33 reported failure to cease smoking, and the smoking cessation rate was $42.4 \%$.

\section{The Predictors of Smoking Cessation}

There was no difference between the cessation success group and the failed group in terms of most factors, including age, education, visits to an outpatient clinic, age of smoking initiation, duration of smoking habit, FTND score, and use of pharmacotherapy. However, we found a statistically significant difference in both cause of admission $(\mathrm{P}=0.039)$ and total number of counseling sessions after discharge $(\mathrm{P}<0.001)$. In the successful smoking cessation group, $54.7 \%$ were patients admitted to the department of neurology, $26.4 \%$ to cardiology, and $18.9 \%$ to pulmonology. In the failure group, $45.8 \%$ were cardiology patients. Although it was not statistically significant, the successful smoking cessation group differed slightly from the failed group in terms of duration of hospitalization. In the successful cessation group, $34.7 \%$ of patients were admitted for 4 days or less, $45.8 \%$ were admitted for 5 to 8 days, and $19.4 \%$ were admitted for 9 days or more. In contrast, these numbers were $18.9 \%, 45.3 \%$, and $35.8 \%$ in the failed group. Total number of counseling sessions showed a meaningful difference between the two groups. In the successful smoking cessation group, everyone had at least 1 followup counseling session after discharge, $17 \%$ had 1 or 2 sessions, and $83.0 \%$ had 3 or more. In the failed group, $40.2 \%$ of patients had no additional counseling after discharge, $36.2 \%$ had 1 or 2 sessions, and only $23.6 \%$ of participants were counseled 3 or more times (Table 1 ).

The multivariate analysis included age, education, cause of admission, duration of hospitalization, total number of counseling sessions, visits to an outpatient clinic, age of smoking initiation, duration of smoking habit, daily consumption, and FTND score. Our goal was to identify the predictors of successful smoking cessation at 3 months. We found that participants with three or more counseling sessions after discharge were more likely to quit smoking than participants without additional counseling (odds ratio [OR], 277.595; 95\% confidence interval [CI], 24.087 to $>999.999$ ). When compared with participants with one or two sessions, the probability of cessation was higher in patients with three or more counseling sessions (OR, 13.118; 95\% CI, 4.018 to 57.400) (Table 2). 
Table 1. Clinical characteristics of participants who succeed and failed to quit smoking for 3 months

\begin{tabular}{|c|c|c|c|c|}
\hline Characteristic & Total $(\mathrm{n}=125)$ & ence succeeded $(n=53)$ & Abstinence failed $(\mathrm{n}=72)$ & P-value \\
\hline Age (y) & $57.89 \pm 10.23$ & $58.20 \pm 10.30$ & $57.66 \pm 10.26$ & 0.772 \\
\hline Education & & & & 0.332 \\
\hline Middle school or less & $41(32.8)$ & $15(28.3)$ & $26(36.1)$ & \\
\hline High school & $45(36.0)$ & $23(43.4)$ & $22(30.6)$ & \\
\hline College or more & $39(31.2)$ & $15(28.3)$ & $24(33.3)$ & \\
\hline Cause of admission & & & & 0.039 \\
\hline Nuerologic disease & $53(42.4)$ & $29(54.7)$ & $24(33.3)$ & \\
\hline Cardiologic disease & $47(37.6)$ & $14(26.4)$ & $33(45.8)$ & \\
\hline Pulmonologic disease & $25(20.0)$ & $10(18.9)$ & $15(20.8)$ & \\
\hline Duration of admission (d) & & & & 0.054 \\
\hline$\leq 4$ & $35(28.0)$ & $10(18.9)$ & $25(34.7)$ & \\
\hline $5-8$ & $57(45.6)$ & $24(45.3)$ & $33(45.8)$ & \\
\hline$\geq 9$ & $33(26.4)$ & $19(35.8)$ & $14(19.4)$ & \\
\hline Total no. of counseling & & & & $<0.001$ \\
\hline 0 (follow-up loss) & $29(23.2)$ & 0 & $29(40.2)$ & \\
\hline $1-2$ & $35(28.0)$ & $9(17.0)$ & $26(36.2)$ & \\
\hline$\geq 3$ & $61(48.8)$ & $44(83.0)$ & $17(23.6)$ & \\
\hline Outpatient department visit & $24(19.2)$ & $14(26.4)$ & $10(13.9)$ & 0.107 \\
\hline Age of smoking initiation (y) & $21.14 \pm 4.76$ & $20.92 \pm 3.97$ & $21.30 \pm 5.29$ & 0.661 \\
\hline Duration of smoking (y) & $35.86 \pm 11.73$ & $36.28 \pm 11.48$ & $35.55 \pm 11.98$ & 0.733 \\
\hline $\begin{array}{l}\text { Daily tobacco consumption } \\
\text { (no. of cigarettes) }\end{array}$ & $23.52 \pm 13.19$ & $21.90 \pm 12.26$ & $24.70 \pm 13.79$ & 0.242 \\
\hline Fagerstrom score & & & & 0.691 \\
\hline$\leq 3$ & $22(17.6)$ & $11(20.8)$ & $11(15.3)$ & \\
\hline $4-6$ & $56(44.8)$ & $22(41.5)$ & $34(47.2)$ & \\
\hline$\geq 7$ & $47(37.6)$ & $20(37.7)$ & $27(37.5)$ & \\
\hline Modalities of intervention & & & & 0.256 \\
\hline Education only & $43(34.4)$ & $15(28.3)$ & $28(38.9)$ & \\
\hline Pharmacotherapy & $82(65.6)$ & $38(71.7)$ & $44(61.1)$ & \\
\hline Nicotine replacement therapy & $58(70.7)$ & $28(73.7)$ & $30(68.2)$ & \\
\hline Varenicline & $23(28.1)$ & $10(26.3)$ & $13(29.5)$ & \\
\hline Bupropion slow release & $1(1.2)$ & 0 & $1(2.3)$ & \\
\hline
\end{tabular}

Values are presented as number $(\%)$ or mean $\pm \mathrm{SD}$. 
Table 2. Predictors of successful cessation for 3 months $^{*}$

\begin{tabular}{|c|c|c|}
\hline Variable & Estimated odds ratio & $95 \%$ confidence interval \\
\hline Age (y) & 1.026 & $0.871-1.209$ \\
\hline \multicolumn{3}{|l|}{ Education } \\
\hline College or more & 1 & \\
\hline High school & 1.554 & $0.447-5.641$ \\
\hline Middle school or less & 0.59 & $0.130-2.720$ \\
\hline \multicolumn{3}{|l|}{ Cause of admission } \\
\hline Pulmonologic disease & 1 & \\
\hline Cardiologic disease & 1.281 & $0.272-6.336$ \\
\hline Nuerologic disease & 4.887 & $1.141-25.299$ \\
\hline \multicolumn{3}{|l|}{ Duration of admission (d) } \\
\hline$\leq 4$ & 1 & \\
\hline $5-8$ & 1.157 & $0.338-3.993$ \\
\hline$\geq 9$ & 3.202 & $0.797-14.423$ \\
\hline \multicolumn{3}{|l|}{ Total no. of counseling } \\
\hline 0 (follow-up loss) & 1 & \\
\hline $1-2$ & 21.161 & $2.020->999.999$ \\
\hline$\geq 3$ & 277.595 & $24.087->999.999$ \\
\hline$\geq 3$ vs. $1-2$ (reference) & 13.118 & $4.018-57.400$ \\
\hline OPD visit & 0.958 & $0.293-3.067$ \\
\hline Age of smoking initiation (y) & 1.07 & $0.866-1.333$ \\
\hline Duration of smoking (y) & 1.003 & $0.861-1.172$ \\
\hline Daily tobacco consumption (no. of cigarettes) & 0.972 & $0.930-1.011$ \\
\hline \multicolumn{3}{|l|}{ Fagerstrom score } \\
\hline$\geq 7$ & 1 & \\
\hline $4-6$ & 1.125 & $0.329-3.857$ \\
\hline$\leq 3$ & 3.007 & $0.577-19.186$ \\
\hline \multicolumn{3}{|l|}{ Modalities of intervention } \\
\hline Pharmacotherapy & 1.184 & $0.425-3.261$ \\
\hline
\end{tabular}

*Results from SAS logistic with firth option.

\section{DISCUSSION}

We conducted a study of inpatient smokers admitted to the departments of neurology, cardiology, and pulmonology in Asan Medical Center from September 2011 to February 2013 in order to identify the effectiveness of an inpatient smoking cessation program along with regular follow-up counseling after discharge.

The 3-month smoking cessation rate was $42.4 \%$ in this study. This is comparable to the results of another study (with a $41.1 \%$ cessation rate) in which 116 German inpatient smokers were enrolled and monitored for 3 months. ${ }^{17)}$ Our study was more cost-effective, with an average of 2.1 counseling sessions compared to eight sessions of telephone counseling in the German study. One reason for the relatively high cessation rate was high motivation. Indeed, the most common cause for a patient's 
decision to quit smoking was health, according to the self-report questionnaire. In this study, $65.6 \%$ of participants were prescribed medication, and this frequent prescription rate might also have contributed to the cessation rate. ${ }^{18)}$

We found that the cessation rate for patients who had three or more counseling sessions after discharge was significantly higher than for patients with zero to two sessions. Regular follow-up counseling may help to increase the cessation rate in several ways. Above all, smokers can discuss and get medical advice about withdrawal symptoms or side effects of pharmacotherapy. Moreover, medical and social support can be provided to smokers in parallel. It also extends the duration of smoking cessation treatment, and medical workers can check the patient's smoking status and motivation regularly. ${ }^{19)}$ In a meta-analysis, not all smoking cessation programs were effective. However, inpatient counseling for 15 minutes and monitoring for more than a month after discharge resulted in a significantly higher cessation rate compared to control groups. There was no significant effect when inpatient counseling sessions were shorter than 15 minutes, or additional counseling sessions lasted for less than 1 month. ${ }^{5)}$ Generally, a longer follow-up duration means more counseling sessions, so this corresponds with the results of our study.

Whereas pharmacotherapy ${ }^{10)}$ and nicotine dependency have been considered useful predictors for smoking cessation in the past, they were not significant predictors in the current study. To explain this outcome, we consider the possibility that patients did not always use the prescribed medication. Smoking cessation medication or aids were prescribed to $65.6 \%$ of all participants, but we could not check whether they used the medication. This outcome may also be due to strong motivation. Inpatient smokers usually have stronger motivation to quit smoking than the general population and are more likely to quit using willpower without pharmacotherapy, so the effectiveness of pharmacotherapy might have been underestimated. ${ }^{20)}$ Lastly, the effectiveness of our intervention program might be strong enough to underestimate the effectiveness of pharmacotherapy. ${ }^{21)}$ We believe the results for nicotine dependence in this study could also be due to strong motivation and the intensive cessation program.

The most important limitation of our study is the fact that smoking cessation was confirmed by selfreport. In other words, the smoking cessation rate may have been overestimated because we did not use any other biochemical methods to confirm smoking status. On the other hand, the smoking cessation rate may have been underestimated, due to missing participants during follow-up. We considered participants who failed to complete follow-up sessions to be cessation failures; those who successfully quit smoking but did not participate in additional counseling would be included in the failure group. The small sample size and the 3-month follow-up duration (relatively short to judge long-term cessation in the general population) are also weaknesses of this study. It is possible that differences in cessation rate are associated with the residents who counseled our inpatient smokers. However, we believe this is of minimal concern, because we educated all residents before they counseled smokers and they all used the same educational materials and questionnaires.

In conclusion, inpatient smoking cessation counseling and regular follow-up counseling for 3 months after discharge contributed to an increase in cessation rate (42.4\%). Participants who were counseled 
three or more times were significantly more likely to quit smoking successfully than participants without further counseling, and the probability of cessation tended to increase with the total number of counseling sessions. On the basis of these results, we conclude that it is important to improve inpatient smoking cessation programs more systematically and consistently.

\section{CONFLICT OF INTEREST}

No potential conflict of interest relevant to this article was reported.

\section{REFERENCES}

1. World Health Organization. Guidelines for controlling and monitoring the tobacco epidemic [Internet]. Geneva: World Health Organization; 1998 [cited 2013 Oct 21]. Available from: http://www.who.int/iris/handle/10665/42049.

2. Jee SH, Yun JE, Park JY, Sull JW, Kim IS. Smoking and cause of death in Korea: 11 years follow-up prospective study. Korean J Epidemiol 2005;27:182-90.

3. Jee SH, Samet JM, Ohrr H, Kim JH, Kim IS. Smoking and cancer risk in Korean men and women. Cancer Causes Control 2004;15:341-8.

4. Anthonisen NR, Skeans MA, Wise RA, Manfreda J, Kanner RE, Connett JE, et al. The effects of a smoking cessation intervention on 14.5-year mortality: a randomized clinical trial. Ann Intern Med 2005;142:233-9.

5. Godtfredsen NS, Vestbo J, Osler M, Prescott E. Risk of hospital admission for COPD following smoking cessation and reduction: a Danish population study. Thorax 2002;57:967-72.

6. Rigotti NA. Smoking cessation in patients with respiratory disease: existing treatments and future directions. Lancet Respir Med 2013;1:241-50.

7. Rigotti NA, Arnsten JH, McKool KM, Wood-Reid KM, Pasternak RC, Singer DE. Smoking by patients in a smoke-free hospital: prevalence, predictors, and implications. Prev Med 2000;31(2 Pt 1):159-66.

8. Rigotti NA, Clair C, Munafo MR, Stead LF. Interventions for smoking cessation in hospitalised patients. Cochrane Database Syst Rev 2012;5:CD001837.

9. France EK, Glasgow RE, Marcus AC. Smoking cessation interventions among hospitalized patients: what have we learned? Prev Med 2001;32:376-88.

10. Regan S, Viana JC, Reyen M, Rigotti NA. Prevalence and predictors of smoking by inpatients during a hospital stay. Arch Intern Med 2012;172:1670-4.

11. Bolman C, de Vries H, van Breukelen G. A minimal-contact intervention for cardiac inpatients: long-term effects on smoking cessation. Prev Med 2002;35:181-92.

12. Hennrikus DJ, Lando HA, McCarty MC, Klevan D, Holtan N, Huebsch JA, et al. The TEAM project: the effectiveness of smoking cessation intervention with hospital patients. Prev Med 2005;40:249-58. 
13. Stevens VJ, Glasgow RE, Hollis JF, Mount K. Implementation and effectiveness of a brief smoking-cessation intervention for hospital patients. Med Care 2000;38:451-9.

14. Smith PM, Corso L, Brown KS, Cameron R. Nurse case-managed tobacco cessation interventions for general hospital patients: results of a randomized clinical trial. Can J Nurs Res 2011;43:98-117.

15. Hajek P, Taylor TZ, Mills P. Brief intervention during hospital admission to help patients to give up smoking after myocardial infarction and bypass surgery: randomised controlled trial. BMJ 2002;324:87-9.

16. Heatherton TF, Kozlowski LT, Frecker RC, Fagerstrom KO. The Fagerstrom Test for Nicotine Dependence: a revision of the Fagerstrom Tolerance Questionnaire. Br J Addict 1991;86:1119-27.

17. Metz K, Floter S, Kroger C, Donath C, Piontek D, Gradl S. Telephone booster sessions for optimizing smoking cessation for patients in rehabilitation centers. Nicotine Tob Res 2007;9:853-63.

18. Hartmann-Boyce J, Stead LF, Cahill K, Lancaster T. Efficacy of interventions to combat tobacco addiction: Cochrane update of 2012 reviews. Addiction 2013;108:1711-21.

19. Brandon TH, Collins BN, Juliano LM, Lazev AB. Preventing relapse among former smokers: a comparison of minimal interventions through telephone and mail. J Consult Clin Psychol 2000;68:103-13.

20. Eisenberg MJ, Grandi SM, Gervais A, O'Loughlin J, Paradis G, Rinfret S, et al. Bupropion for smoking cessation in patients hospitalized with acute myocardial infarction: a randomized, placebo-controlled trial. J Am Coll Cardiol 2013;61:524-32.

21. Smith PM, Burgess E. Smoking cessation initiated during hospital stay for patients with coronary artery disease: a randomized controlled trial. CMAJ 2009;180:1297-303. 